\title{
Structure of a proteolytically resistant core from the severe acute respiratory syndrome coronavirus $\mathbf{S 2}$ fusion protein
}

\author{
Vinit M. Supekar, Chiara Bruckmann, Paolo Ingallinella, Elisabetta Bianchi, Antonello Pessi, and Andrea Carfí ${ }^{\ddagger}$ \\ Istituto di Ricerche di Biologia Molecolare "P. Angeletti," Via Pontina Km 30,600, 00040 Pomezia (Rome), Italy
}

Edited by Stephen C. Harrison, Harvard Medical School, Boston, MA, and approved November 10, 2004 (received for review August 19, 2004)

\begin{abstract}
A coronavirus (CoV) has recently been identified as the causative agent of the severe acute respiratory syndrome (SARS) in humans. CoVs enter target cells through fusion of viral and cellular membranes mediated by the viral envelope glycoprotein $\mathrm{S}$. We have determined by $\mathrm{x}$-ray crystallography the structure of a proteolytically stable core fragment from the heptad repeat (HR) regions HR1 and HR2 of the SARS-CoV S protein. We have also determined the structure of an HR1-HR2 S core fragment, containing a shorter HR1 peptide and a C-terminally longer HR2 peptide that extends up to the transmembrane region. In these structures, three HR1 helices form a parallel coiled-coil trimer, whereas three HR2 peptides pack in an oblique and antiparallel fashion into the coiled-coil hydrophobic grooves, adopting mixed extended and $\alpha$-helical conformations as in postfusion paramyxoviruses $F$ proteins structures. Our structure positions a previously proposed internal fusion peptide adjacent to the N-terminus of HR1. Peptides from the HR2 region of SARS-CoV S have been shown to inhibit viral entry and infection in vitro. The structures presented here can thus open the path to the design of small-molecule inhibitors of viral entry and candidate vaccine antigens against this virus.
\end{abstract}

coiled-coil | membrane fusion | viral entry | heptad repeat

Coror oronaviruses (CoVs) are enveloped positive-stranded RNA viruses that infect avian and mammalian species, generally causing respiratory, enteric, and neurological diseases in their hosts $(1,2)$. Recently, another CoV was identified that infects humans causing an atypical and often lethal pneumonia known as severe acute respiratory syndrome (SARS) (3-6). This virus is not related to other previously characterized human or animal CoVs and forms a distinct phylogenetic group within the genus (7).

As for other enveloped viruses, entry of CoVs into target cells requires fusion between the viral and cellular membranes. Recent evidence suggests that entry of SARS-associated CoV (SARS-CoV) is mediated by the Spike (S) envelope glycoprotein through a $\mathrm{pH}$-dependent endocytic pathway $(8,9)$. Similar to other viral membrane fusion proteins, $\mathrm{S}$ can be subdivided into two domains, S1 and S2, which, for some CoVs, but not for SARS-CoV, are formed after proteolytic cleavage (Fig. 1A). S1 mediates binding to the receptor which, for the SARS-CoV, has recently been identified as the angiotensin-converting enzyme 2 $(10,11)$. Analysis of CoV S2 sequences shows the presence of two 4-3 heptad repeats (HRs) of hydrophobic residues, HR1 and HR2, typical of coiled coils (12), separated by an $\approx 170$-aa-long intervening domain (Fig. 1) (13). The presence of conserved HRs in S2 suggests, in analogy with other virus-encoded class I fusion glycoproteins, that this region is directly involved in the membrane fusion process (14).

Influenza virus hemagglutinin (HA) is the best characterized class I viral fusion protein. Structures of HA have been determined for the uncleaved, the cleaved metastable HA1-HA2 heterodimer, and the postfusion conformation (see ref. 15 for a review). HA1 mediates receptor binding, whereas HA2, at low endosomal $\mathrm{pH}$, undergoes a series of conformational changes that are required for membrane fusion. At the end of the conformational changes, the membrane-spanning region and the fusion peptide, at the $\mathrm{C}$ and $\mathrm{N}$ terminus of $\mathrm{HA} 2$, respectively, are in close proximity as a result of the formation of a trimer of hairpins structure. This structure is thought to form concomitantly or just after membrane fusion and is generally referred to as "postfusion" or "fusion-active" conformation. Structures of the postfusion conformation have been obtained for other viral class I fusion proteins $(14,15)$, all pointing to the importance of the formation of the trimer of hairpins structure and of the juxtaposition of the transmembrane (TM) region with the fusion peptide for the membrane fusion process.

Peptides from the HR2 region of the HIV-1 and simian immunodeficiency virus gp41, of simian parainfluenza virus $5 \mathrm{~F}$, and other class I fusion proteins, have been shown to be inhibitors of viral entry. In the case of HIV-1, a 36-mer HR2peptide (T20/fuzeon) is currently used in the treatment of HIV-1-infected patients (16). For HIV-1 gp41, HR2 peptides are thought to function by binding to the transiently exposed HR1 coiled-coil trimer and blocking the formation of the HR1-HR2 six-helical bundle (17). For the murine CoV mouse hepatitis virus (MHV), HR2 peptides have also been shown to inhibit entry at nanomolar concentrations (18). However, the corresponding peptides from SARS-CoV inhibited viral entry in Vero cells with an $\mathrm{IC}_{50}$ in the micromolar range $(19,20)$. This difference in inhibition could be due to a weaker interaction between the HR1 and HR2 regions of SARS-CoV S2 (19), but it cannot be excluded that other factors such as the kinetics of membrane fusion or differences in the entry pathway of these two viruses could also affect the inhibitory properties of HR2 peptides.

We have previously shown (21) that peptides including the entire HR1 (residues 889-972) and HR2 (residues 1142-1185) regions of SARS-CoV S2 form a trimeric, proteolytically stable, $\alpha$-helical core complex. This complex has now been crystallized and its structure solved at $1.6-\AA$ resolution. In the course of a study aimed at characterizing the interaction between peptides from the HR1 and HR2 regions of SARS-CoV S2, we also crystallized and solved the structure of an HR1-HR2 complex containing a shorter HR1 peptide (N3:914-949) and an HR2 peptide (C2:1149-1193) that extends at the $C$ terminus to the beginning of the TM region.

The structures of the SARS-CoV S2 fusion protein core presented here provides a framework for the design of entry inhibitors that could be used in the therapeutic intervention against this virus. Moreover, these structures can guide the design of candidate antigens for an anti-SARS vaccine.

This paper was submitted directly (Track II) to the PNAS office.

Abbreviations: CoV, coronavirus; HR, heptad repeat; SARS, severe acute respiratory syndrome; S, Spike; HA, hemagglutinin; MHV, mouse hepatitis virus; TM, transmembrane.

Data deposition: The atomic coordinates and structure factors have been deposited in the Protein Data Bank, www.pdb.org (PDB ID codes 2BEQ and 2BEZ).

*To whom correspondence should be addressed. E-mail: andrea_carfi@merck.com.

C 2004 by The National Academy of Sciences of the USA 


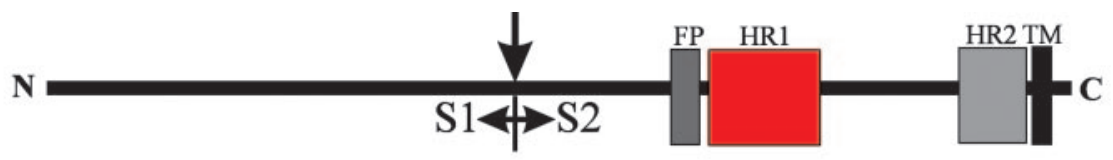

B

HR1 region

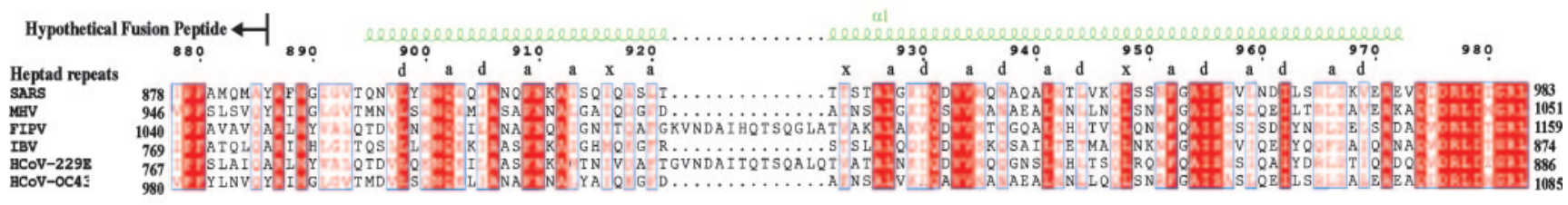

HR2 region

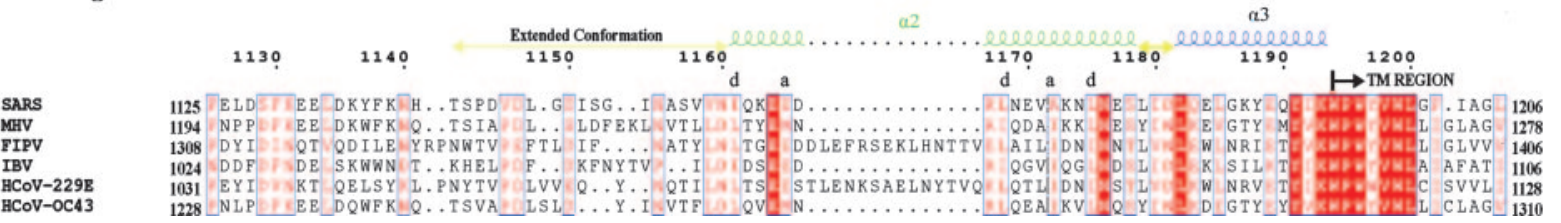

Fig. 1. Organization and sequence alignments of CoV S HRs. $(A)$ Schematic organization of CoVs $S$. The cleavage site in the related MHV $S$ is shown. (B) Sequence alignment for the HR regions for CoV S2 proteins. Hydrophobic residues with a, d, and x packing in the HR1 HRs are indicated above the sequence.

\section{Materials and Methods}

Preparation of HR1-HR2 Complexes and Crystallization. Preparation and purification of synthetic and recombinant $\mathrm{C}$ and $\mathrm{N}$ peptides from the HR regions of SARS-CoV S2 and of the HR1-HR2 proteolytically resistant core have been reported (21). MS analysis of the proteinase K-resistant SARS-CoV S2 core complex showed some heterogeneity at the $\mathrm{N}$ terminus of HR1 and at the $\mathrm{C}$ terminus of HR2 (Fig. 6, which is published as supporting information on the PNAS web site). The HR1 fragments present in the complex correspond to residues 891972, 897-972, and 901-972, with the second fragment being the most abundant. The HR2 peptides start at residue 1142 and end at residues 1184 and 1185 .

Crystals of the SARS-CoV S2 core were grown by vapor diffusion in hanging drops combining 1:1 volume of protein (25 $\mathrm{mg} / \mathrm{ml}$ in $20 \mathrm{mM}$ Tris $\cdot \mathrm{HCl}, \mathrm{pH} 7.5 / 100 \mathrm{mM} \mathrm{NaCl}$ ) and reservoir solution (1.8 $\mathrm{M}$ ammonium sulfate $/ 100 \mathrm{mM}$ Tris $\cdot \mathrm{HCl}, \mathrm{pH} 7)$. The crystals belong to the $\mathrm{H} 32$ space group with cell parameters $a=b=37.345 \AA$, and $c=469.832 \AA$, and contain one HR1-HR2 heterodimer in the asymmetric unit. The N3/C2 complex was prepared by dissolving equimolar amounts of N3 and C2 peptides in $10 \mathrm{mM}$ PBS, $\mathrm{pH}$ 7. The resulting complex $(10 \mathrm{mg} / \mathrm{ml})$ was then crystallized in $30 \%$ polyethylene glycol $8000 / 100 \mathrm{mM}$ sodium cacodylate, $\mathrm{pH} 6.5 / 200 \mathrm{mM}$ sodium acetate. The N3/C2 crystals belong to the $\mathrm{C} 2$ space group with cell parameters $a=$ $99.907 \AA, b=42.903 \AA, c=59.826 \AA$, and $\beta=116.69^{\circ}$ and contain one $\mathrm{N} 3 / \mathrm{C} 2$ trimer in the asymmetric unit.

Data Collection, Structure Determination, and Refinement. Highresolution native data sets for the SARS-CoV S2 core and for the N3/C2 complex, respectively, were collected at the ID14 beamline at the European Synchrotron Radiation Facility (Grenoble, France). Before data collection, crystals were cryoprotected with $20 \%$ glycerol and then flash-frozen in liquid nitrogen. All of the data were processed with DENZO and scaled with SCALEPACK (22) (Table 1, which is published as supporting information on the PNAS web site). For structure determination purposes, Val-1159, which was predicted to be exposed in the postfusion complex, was mutated to cysteine, and the mutant complexes were crystallized as the corresponding native ones, except for the addition of 5 mM DTT. For derivatization, crystals of the mutant complexes were soaked in presence of $100 \mu \mathrm{M} \mathrm{HgAc}$ for $12 \mathrm{~h}$ before data collection. The derivative data were collected in the laboratory on a rotating anode x-ray generator and a MAR345 image plate detector. The two structures were solved independently by using the single isomorphous replacement with anomalous scattering method, followed by density modification as implemented in CNS (23). Several cycles of model building by using O (24) and map improvement using ARP/WARP (25) in MOLREP mode allowed building the complete models. The SARS-CoV S2 core and the N3/C2 structures have been refined to $1.6-\AA$ resolution by using REFMAC (26) with a maximum likelihood target and individual $B$-factor refinement. Anisotropic scaling and bulk solvent correction were applied throughout. Water molecules were added at the end of the refinement in SIGMAA-weighted $\left(F_{\mathrm{o}}-F_{\mathrm{c}}\right)$ electron density maps by using a 3- $\sigma$ cutoff. The final $R_{\text {free }}$ values for the two structures are $23.8 \%$ and $24.2 \%$, respectively (Table 1 ). In the SARS-CoV S2 core structure, no interpretable electron density was observed for the first four residues of HR1 (residues 891-894) or for the last five C-terminal residues of HR2 (residues 1181-1185).

\section{Results}

A Long Trimeric Coiled-Coil HR1 Structure. The SARS-CoV S2 core adopts an elongated structure, $\approx 115 \AA$ in length and $30 \AA$ in diameter. Three parallel HR1 peptides form an inner trimeric coiled coil, with three HR2 peptides packing in a left-handed and antiparallel direction into its grooves (Fig. $2 A$ ). This antiparallel orientation of the HR1 and HR2 regions would bring the TM region of $\mathrm{S} 2$ the $\mathrm{C}$ terminus of $\mathrm{HR} 2$ in spatial proximity to a hypothetical internal fusion peptide region N-terminal to HR1 (see below). These characteristics are consistent with a postfusion structure.

The majority of the HR1 amino acid side chains in the hydrophobic a and d positions of the HRs $(\mathrm{a}-\mathrm{g})$ assume a classical "knobs-into-holes" close packing typical of coiled coils (27). There are, however, irregularities in both the HRs and in the packing of internal hydrophobic residues (Figs. $1 B$ and $2 B$ ). One such irregularity, between residues 941 and 955, is a 3-4-4-3 repetition ("stutter") instead of the usual 4-3-4-3 repetition; Leu-948 occupies an $\mathrm{x}$ position, with its side chain pointing 


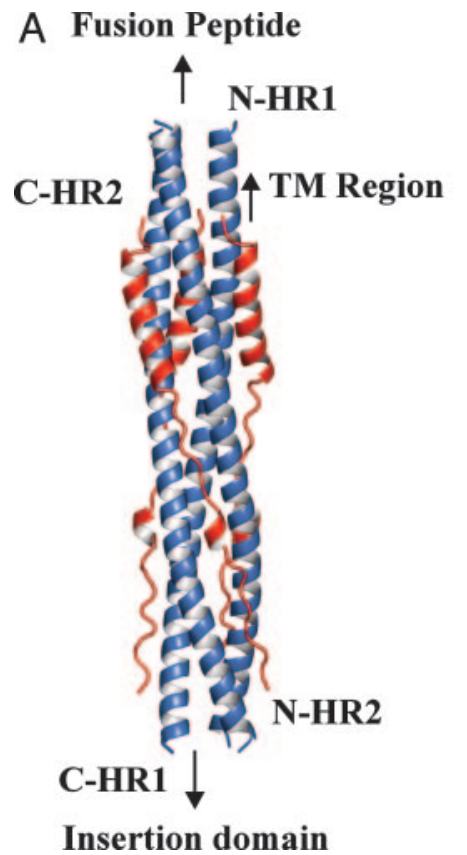

B

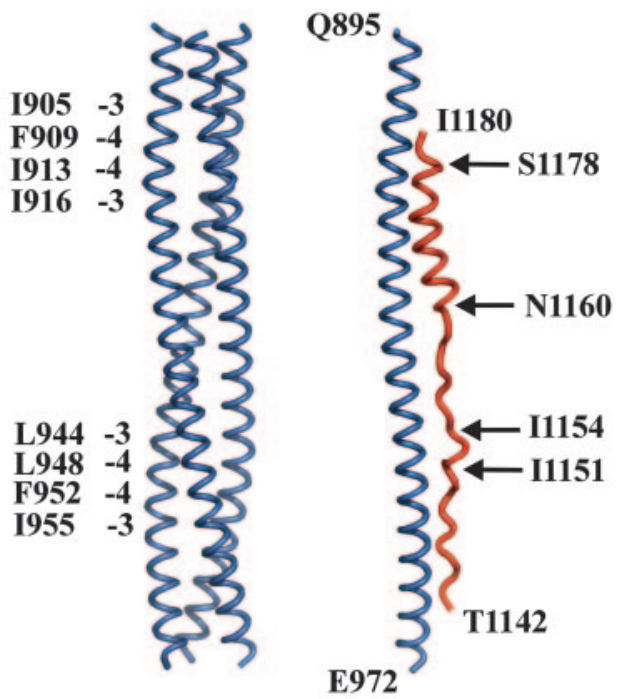

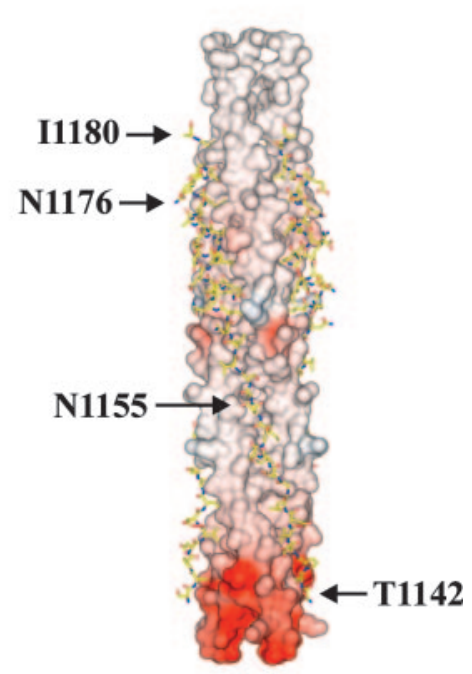

\section{Insertion domain}

Fig. 2. Structure of the SARS-CoV S2 core. (A) Ribbon representation of the $S 2$ core structure. $(B)$ Irregularity in the 3-4 repetition of the HR1 trimer. (C) An HR1-HR2 heterodimer. HR1 is blue and HR2 is red. $(D)$ Electrostatic potential surface of the HR1 inner coil trimer with HR2 peptides shown as a ball-and-stick representation packing on the trimer grooves; two conserved glycosylation sites predicted at N1155 and N1176 of HR2 are indicated.

toward the threefold axis (28). Another 3-4-4-3 pattern is present at the $\mathrm{N}$ terminus of HR1 between residues 902 and 916. In this region and in its proximity, deviations from the classical knobs-into-holes packing are observed: Ile-916 and Thr-923 adopt an $\mathrm{x}$ packing, whereas Ile-913 and Leu-920 adopt a nonclose packing (28), leading to the formation of a hole along the trimer axis (Fig. $7 A$ and $B$, which is published as supporting information on the PNAS web site) and unwinding of the coiled-coil $\mathrm{N}$ terminus. Sequence alignments show that with the exception of the position corresponding to Leu-920 in SARSCoV S2, these packing irregularities are likely to be present in all members of the CoV family (Fig. $1 B$ ).

Three conserved polar residues occupy internal positions in the trimeric core: Gln-902 in an a position at the $\mathrm{N}$ terminus of HR1, and Thr-923 and Asn-937 in the middle of the trimer in $x$ and d positions, respectively. The side chain of Asn-937 points outward toward the HR2 peptides, generating a prominent internal water-filled cavity (Fig. $7 C$ ). Polar residues and irregularities in coiled coils are found in other viral fusion proteins and have been proposed to have a role in the conformational changes that drive fusion. In influenza HA, a threonine is present at the site of the loop-to-helix transition associated with the low $\mathrm{pH}$ conformational change (29). Thus, in CoVs, Thr-923 or Asn-937 could occupy a similar position in the prefusion conformation of the $\mathrm{S}$ protein.

A Mixed Extended Helical HR2 Structure. A total of $38 \mathrm{HR} 2$ residues, in both extended and helical conformations, span $\approx 80 \AA$, matching most of the length of the HR1 trimer (Fig. $2 B$ and $C$ ). Between the N-terminal Thr-1142 and Asn-1160, HR2 has an extended conformation, matching HR1 residues 962-929 (Fig. 2 $C$ and $D$ ). Many hydrogen-bond interactions between side chains of polar HR1 amino acids and main-chain nitrogen and oxygen atoms of HR2 constrain the conformation of this extended part of HR2; side chains of hydrophobic HR2 residues (Val-1146, Leu-1148, Ile-1151, Ile-1154, Ala-1156, Val-1159, and Ile-1161) also insert into hydrophobic pockets in the trimer grooves. One such pocket, filled by the side chains of Leu-1148 and Val-1146
(Fig. 3), could be a suitable target for binding of a smallmolecule entry inhibitor, although it is not as prominent as those observed in HIV-1 gp41 $(30,31)$ and simian parainfluenza virus $5 \mathrm{~F}(32)$.

From Ile-1161 to Ser-1178, HR2 forms an amphipathic $\alpha$ helix, which packs against the HR1 trimer grooves of residues 909-928. The HR2 helix is oblique with respect to the HR1 trimer axis and does not form a classical coiled-coil-like interaction with the HR1 helices, as observed for the $\mathrm{C} / \mathrm{N}$ peptide packing of HIV-1 gp41 $(30,31)$ and Ebola Gp2 $(33,34)$. The interaction with the HR1 trimer is mainly hydrophobic, with apolar amino acids of the HR2 helix (Ile-1161, Leu-1168, Val-1171, and Leu-1175) packing against the HR1 trimer grooves and only three interactions formed by charged/polar residues. After residue 1178, the HR2 chain again assumes an extended conformation for two additional amino acids. Thus, the

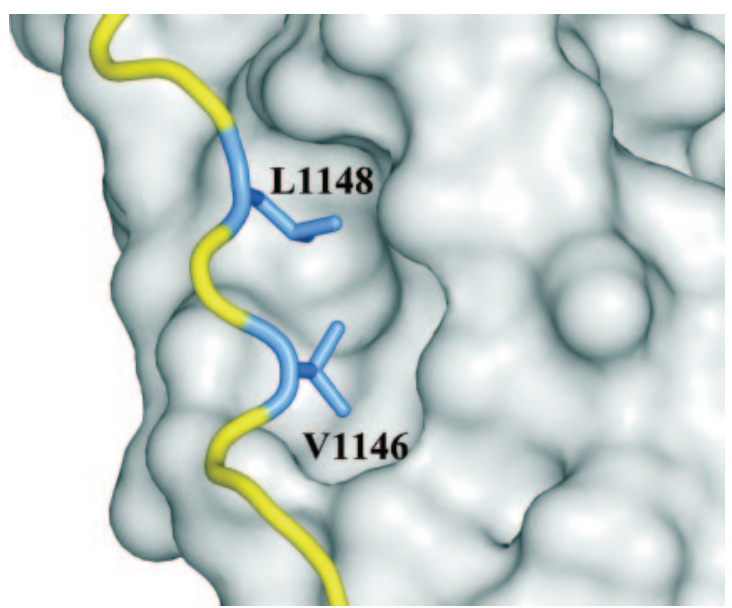

Fig. 3. A hydrophobic pocket on the surface of the HR1 trimer with residues V1146 and L1148 from HR2 pointing toward it. 
last visible residue is Ile-1180, leaving $\approx 10$ residues at the $\mathrm{N}$ terminus of HR1 exposed to solvent (Fig. $2 C$ and $D$ ).

Each HR2 peptide buries a total surface of $3,600 \AA^{2}$ on an HR1 trimer groove; $1,900 \AA^{2}$ are buried by the extended part of HR2 and 1,700 $\AA^{2}$ are buried by the helical part, suggesting an important contribution of both regions to the stabilization of the $\mathrm{S} 2$ postfusion structure and to the membrane fusion process. Residues at both the $\mathrm{N}$ terminus of HR1 and at the $\mathrm{C}$ terminus of HR2 display increasing thermal parameters, reaching values $>60 \AA^{2}$ for 23 residues at the $N$ terminus of HR1 (the average $B$ factors are $\approx 30 \AA^{2}$ for the remaining 50 C-terminal residues) and for the last 10 residues at the $\mathrm{C}$ terminus of HR2. These high thermal parameters probably reflect a significant flexibility in the membrane-proximal region of the HR1-HR2 complex.

Comparison with MHV S2 Postfusion Core. The $2.5-\AA$ resolution $\mathrm{x}$-ray structure of a somewhat smaller bacterially expressed fragment from the MHV S2 protein has been published (35). The SARS-CoV S2 stable core studied here has an HR1 $\approx 20$ residues longer and an HR2 4 residues longer than the corresponding segments in the MHV S2 crystals. Overall, the two structures are similar, with the majority of the residues occupying hydrophobic HR positions in HR1 either conserved or replaced by conservative mutations. The most prominent change in HR1 is at Ser-919-Leu-920 in SARS-CoV S2, which is replaced by Gly-Phe in MHV S2 with the Phe conserved in all others CoV S proteins (Figs. $1 B$ and $4 A$ ). Mutation of Phe with the smaller Leu generates a void inside the coiled coil that may contribute to the lower thermal stability of the SARS-CoV HR1-HR2 complex relative to MHV (19).

If the HR1 trimer of MHV S2 is superimposed on the corresponding residues of SARS-CoV S2, the rms deviation for $150 \mathrm{HR} 1 \mathrm{C}^{\alpha}$ atoms is only $0.37 \AA$. However, the MHV HR2 helices are rotated $1-2^{\circ}$ in a counterclockwise direction with respect to the SARS-CoV HR2, with average distances of 1.5-2.5 $\AA$ between the $\mathrm{C}^{\alpha}$ positions (Fig. $4 B$ ). The hydrophobic character of the interaction between HR1 and HR2 is conserved in MHV and SARS-CoV S proteins, but sequence changes in HR2 residues at the HR1-HR2 interface are likely to cause the observed shift in the relative positions of the $\alpha$-helices and are mostly responsible for the $350-\AA^{2}$ difference in buried surface between the HR2-HR1 groove interfaces of $\operatorname{MHV}\left(3,420 \AA^{2}\right)$ and SARS-CoV (3,070 $\left.\AA^{2}\right)$ (Fig. 1B). Moreover, deeper pockets on the HR1 grooves and/or bulkier side chains on the HR2 residues, pointing into them are observed in a few positions of the MHV S2 core. For instance, Ala- 1156 in SARS-CoV is a Val in MHV pointing to a conserved pocket, SARS-CoV Val-1159 and Ile-1161 are both Leu in MHV, which fills a deeper pocket in MHV due to a Leu-to-Ile sequence difference at position 930 of SARS HR1. Finally Leu-1175 is conserved but makes a closer contact with the MHV HR1 trimer groove.

A Helical Membrane-Proximal Region. Sequence analysis of CoV S proteins revealed the presence of a hydrophobic Gly- and Ala-rich sequence (residues 857-885) located $\approx 10$ aa upstream of the first N-terminal HR1 residue visible in the structure (Fig. $1 B)$. This sequence, in analogy with other viral fusion proteins, has been proposed to be part of a fusion peptide $(12,19)$. Unlike most class I fusion peptides, this sequence is internal rather than at the $\mathrm{N}$ terminus to $\mathrm{S} 2$. Indeed, there is some evidence that the SARS-CoV S protein remains uncleaved (Fig. $1 A$ and ref. 8). The assignment of this internal sequence to the fusion peptide is consistent with the $\mathrm{x}$-ray model, because approximately the same number of residues, 9 and 14 respectively, would separate the last HR1 and HR2 residues visible in the structure from the membrane $(12,19)$. Whereas the $\mathrm{x}$-ray model of the SARS-CoV S2 postfusion core does not reveal the structure assumed by HR1 and HR2 in proximity of the membrane, we obtained some
A
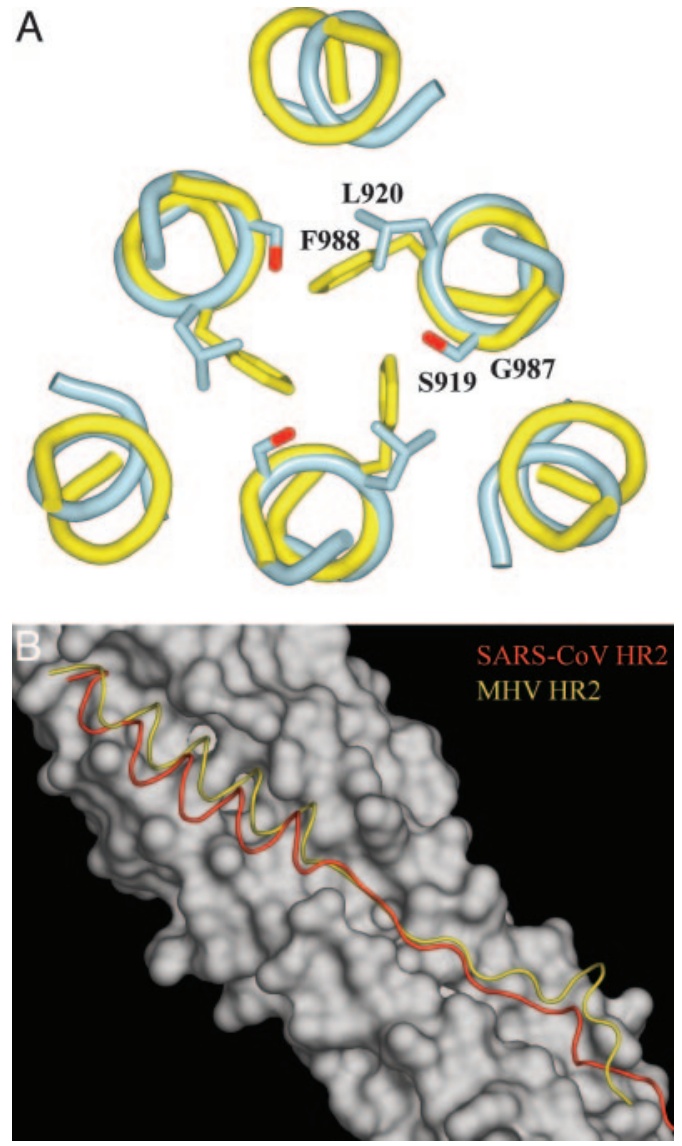

Fig. 4. Differences between MHV and SARS-CoV S2 core structures. $(A)$ Mutation of the conserved Phe-981 of MHV (yellow) with Leu-920 in SARS-CoV S2 (blue). (B) Shift of the HR2 $\alpha$-helices positions between MHV (yellow) and SARS-CoV S2 (red) after superposition of the HR1 trimer.

insight into the structure of this region from the crystal structure of a smaller trimeric S2 fragment composed by the shorter peptides N3 (HR1, 914-949) and C2 (HR2, 1149-1193) (Fig. $5 A$ ). In the $\mathrm{N} 3 / \mathrm{C} 2$ complex, the part of the molecule in common with the proteolytically stable $\mathrm{S} 2$ core fragment adopts an almost identical trimeric structure. The "extra" C-terminal residues of C2 (residues 1182-1193) extend beyond the trimer core (Fig. 5 $B$ and $C$ ). The initial residues in this segment assume an extended conformation (residues 1179-1182, 1179-1181, and 1175-1182 in the three chains), forming a three-stranded $\beta$-sheet almost perpendicular to the HR1 trimer axis. The $\mathrm{C}$-terminal part of the overhang is $\alpha$-helical. The three HR2 C termini point away from the HR1 trimer axis at a distance of $\approx 20-25 \AA$ from each other and make crystal packing contacts with neighboring molecules. If modeled onto the structure of the SARS-CoV S2 core, the extended part of C2 (residues 1179-1183) would run along the HR1 region of the core, as in the MHV S2 core structure, whereas the remaining $10 \mathrm{C}$-terminal residues could pack in a helical conformation on the grooves of the HR1 trimer. This model assumes that HR1 extends in a trimeric coiled-coil structure up to approximately residue 888 , positioning the fusion peptide and the TM region close in space (Fig. 5D). In agreement with this model, limited proteolysis experiments $(19,21)$ have shown that some of these residues, missing in the HR1-HR2 core structure presented here (from residues 1181 to 1193 of HR2 and from residues 889 to 894 of HR1) are at least partially resistant to proteinase K cleavage, suggesting that they may be assembled in the final S2 postfusion structure. 
A

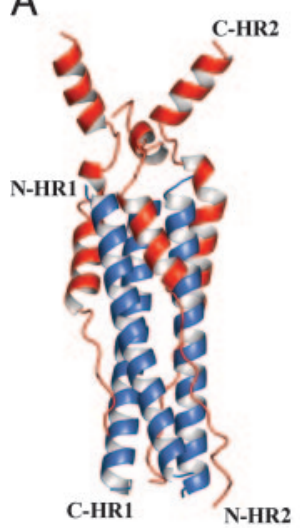

C

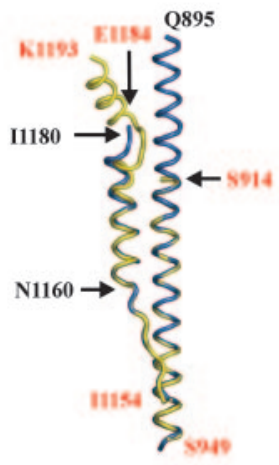

B

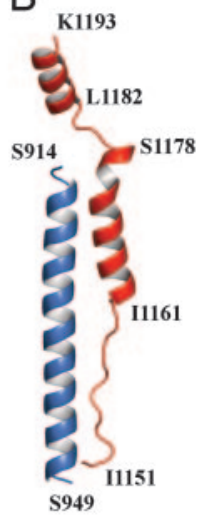

D

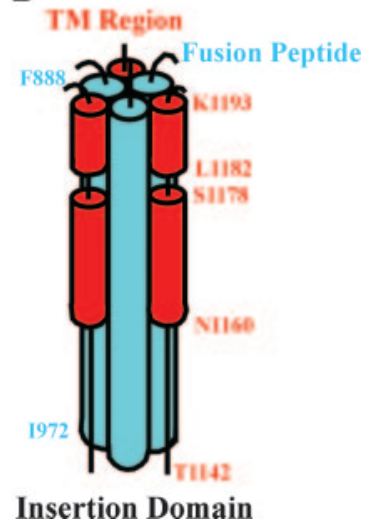

Fig. 5. Ribbon representations of the N3/C2 complex and model of the postfusion structure of SARS-CoV S2. (A) The N3/C2 trimer. N3 is blue and C2 is red $(B)$ An N3/C2 heterodimer, with highlighted "start and end" positions of secondary structure element. $(C)$ Superposition between a HR1-HR2 heterodimer and a N3/C2 heterodimer. (D) Schematic representation of the proposed S2 postfusion structure.

\section{Discussion}

The structure of SARS-CoV S2 core presented here shows several features typical of class I viral fusion proteins. The HR1 region forms a long trimeric helical coiled-coil structure with peptides from the HR2 region packing in an oblique antiparallel manner on the grooves of the HR1 trimer in a mixed extended and helical conformation. Packing of the helical parts of HR2 on the HR1 trimer grooves and formation of a six-helical bundle likely plays an important role in the formation of a stable postfusion structure, similar to that observed for HIV-1 gp41. Moreover, a contribution to HR1HR2 interaction comes from the extended region of HR2, which buries a large surface on the grooves of the HR1 trimer. Similar extended $\mathrm{C}$ peptide regions are present in influenza virus HA2 and paramyxovirus $\mathrm{F}$ and have been shown to have important roles in the membrane fusion process mediated by these two proteins $(36,37)$. In the SARS-CoV S2 core structure, residues toward the $\mathrm{N}$ terminus of HR1 display increasing thermal parameters, reaching values $>60 \AA^{2}$. Although this flexibility could simply result from the lack of interactions with $\mathrm{C}$-terminal residues of the HR2 peptides not present in this structure (Fig. $2 A$ ), we favor the interpretation that the higher thermal parameters reflect an intrinsically lower propensity of the SARS-CoV HR1 N-terminal region to form a stable trimeric coiled-coil structure, probably due to the lack of a closely packed core in this part of HR1. This interpretation is in agreement with our previous study (21) of

a series of partially overlapping HR1 peptides, showing a higher propensity of the C-terminal part of HR1 to form a trimeric coiled coil. Lower stability of the N-terminal region of the HR1 trimer and increased flexibility of HR1 in proximity of the fusion peptide could be requirements for the membrane merging activity of this protein; the lower stability might also indicate that this region folds differently in the prefusion conformation of S2, forming a trimeric coiled coil only in the postfusion structure.

In the structure of the $\mathrm{S} 2$ core formed by the $\mathrm{N} 3$ and $\mathrm{C} 2$ peptides, we find that the last $10 \mathrm{C}$-terminal residues of HR2 assume a $\alpha$-helical conformation. These residues could form $\alpha$-helices both in the native prefusion and in the final postfusion structures of S2. In the latter, they could interact with the trimeric HR1 coiled coil and form a six-helix bundle extending to the surface of the membrane. The interaction between these last few amino acids of HR1 and HR2 in the final postfusion conformation of S2 could release additional free energy and contribute to the membrane fusion process. Interestingly, a peptide that includes the final C-terminal residues of HR2 (up to residue 1193) is a better inhibitor of viral entry than a 5-aa C-terminally shorter peptide (19), supporting the relevance of the interaction between the $\mathrm{N}$ and $\mathrm{C}$ termini of the HRs in the postfusion structure. In this respect, the N3/C2 structure, with the three HR2 C termini (and, therefore, the TM regions), pointing away from the HR1 trimer axis, could resemble an intermediate structure adopted by $\mathrm{S} 2$ at the early stages of the fusion process or before the formation of the final trimer of hairpins.

Among the virus-encoded class I fusion proteins, SARS-CoV $\mathrm{S} 2$ shows the closest structural similarity with paramyxovirus $\mathrm{F}$ proteins (Fig. 8, which is published as supporting information on the PNAS web site). The similarity is due to the length of the HR1 regions ( 83 aa in $F$ and 86 aa in S2), the presence of a long extended region in the membrane distal region of the $\mathrm{C}$ peptide, and the large insertion between the HR regions (210 aa in paramyxovirus $\mathrm{F}$ and 170 aa in $\mathrm{CoV}$ S2). Moreover, in both $\mathrm{CoV}$ $\mathrm{S} 2$ and simian parainfluenza virus $5 \mathrm{~F}$, the fusion peptide may be part of the HR1 coiled coil and protrude into the membrane in a helical conformation (32). These structural similarities raise the possibility that paramyxovirus $\mathrm{F}$ and CoV S2 undergo similar conformational changes to mediate membrane fusion. The prefusion structure of S2 and mutational data will ultimately be needed to dissect the mechanism used by CoVs to bring the viral and cell membranes in proximity.

Peptides derived from the HR2 regions of MHV S2 are nanomolar inhibitors of viral entry. By contrast, HR2 peptides from the SARS-CoV only inhibit virus entry at micromolar concentrations $(19,20)$. Based on the observed lower stability of the SARS-CoV S2 postfusion core relative to the MHV S2 core, it was proposed that the low potency of HR2 peptides could be due to a lower affinity of these peptides for the corresponding HR1 trimer (19). Analysis of the SARS-CoV S2 core structure and the comparison with the MHV S2 core are consistent with this hypothesis. The HR2 peptides pack in a slightly different mode on the otherwise structurally conserved HR 1 trimer because of differences in interface residues in both HR1 and HR2, and a larger surface is buried in the HR1-HR2 interface of MHV S2 than in SARS-CoV S2. Moreover, larger pockets are present in the MHV HR1 grooves and are filled by HR2 residues with larger side chains compared with SARSCoV S2.

In conclusion, we have shown that the SARS-CoV fusion core shares similarities with other class I fusion proteins, and with paramyxovirus $\mathrm{F}$ in particular. The high-resolution structure of the SARS-CoV S2 core here reported has revealed that the CoVs S2 HR1 coiled-coil trimer extends further at both its $\mathrm{N}$ and $\mathrm{C}$ termini than the published MHV S2 structure. Moreover, the 
structure of the N3/C2 complex has shown that the extreme $\mathrm{C}$ terminus of HR2, missing in both SARS-CoV and MHV S2 core structures, folds as an $\alpha$-helix, suggesting that this region may help drive the final stages of membrane fusion.

Finally, two recent studies have reported, for both HIV-1 and human T-lymphotrophic virus 1, that antibodies directed against the N-terminal trimeric coiled coil of gp41 and gp21, respectively, are potent inhibitors of viral cell fusion $(38,39)$. The structure presented here could thus help in the design of

1. Holmes, K. V. (2001) Fields Virology (Lippincott, Williams and Wilkins, New York).

2. Lai, M. M. C. \& Holmes, K. V. (2001) Fields Virology (Lippincott, Williams and Wilkins, New York)

3. Peiris, J. S., Yuen, K. Y., Osterhaus, A. D. \& Stohr, K. (2003) N. Engl. J. Med. 349, 2431-2441.

4. Peiris, J., Lai, S., Poon, L., Guan, Y., Yam, L., Lim, W., Nicholls, J., Yee, W., Yan, W. \& Cheung, M. (2003) Lancet 361, 1319-1325.

5. Ksiazek, T. G., Erdman, D., Goldsmith, C. S., Zaki, S. R., Peret, T., Emery, S., Tong, S., Urbani, C., Comer, J. A., Lim, W., et al. (2003) N. Engl. J. Med. 348, 1953-1966.

6. Fouchier, R. A., Kuiken, T., Schutten, M., van Amerongen, G., van Doornum, G. J., van den Hoogen, B. G., Peiris, M., Lim, W., Stohr, K. \& Osterhaus, A. D. (2003) Nature 423, 240.

7. Rota, P. A., Oberste, M. S., Monroe, S. S., Nix, W. A., Campagnoli, R., Icenogle, J. P., Penaranda, S., Bankamp, B., Maher, K., Chen, M.-H., et al. (2003) Science 300, 1394-1399.

8. Simmons, G., Reeves, J. D., Rennekamp, A. J., Amberg, S. M., Piefer, A. J. \& Bates, P. (2004) Proc. Natl. Acad. Sci. USA 101, 4240-4245.

9. Yang, Z. Y., Huang, Y., Ganesh, L., Leung, K., Kong, W. P., Schwartz, O., Subbarao, K. \& Nabel, G. J. (2004) J. Virol. 78, 5642-5650.

10. Li, W., Moore, M. J., Vasilieva, N., Sui, J., Wong, S. K., Berne, M. A. Somasundaran, M., Sullivan, J. L., Luzuriaga, K., Greenough, T. C., et al. (2003) Nature 426, 450-454.

11. Wong, S. K., Li, W., Moore, M. J., Choe, H. \& Farzan, M. (2003) J. Biol. Chem. 279, 3197-3201.

12. Chambers, P., Pringle, C. R. \& Easton, A. J. (1990) J. Gen. Virol. 71, 3075-3080.

13. de Groot, R. J., Luytjes, W., Horzinek, M. C., van der Zeijst, B. A., Spaan, W. J. \& Lenstra, J. A. (1987) J. Mol. Biol. 196, 963-966.

14. Colman, P. M. \& Lawrence, M. C. (2003) Nat. Rev. Mol. Cell Biol. 4, 309-319.

15. Skehel, J. J. \& Wiley, D. C. (2000) Annu. Rev. Biochem. 69, 531-569.

16. LaBonte, J., Lebbos, J. \& Kirkpatrick, P. (2003) Nat. Rev. Drug Discov. 2, 345-346.

17. Eckert, D. M. \& Kim, P. S. (2001) Annu. Rev. Biochem. 70, 778-810.

18. Bosch, B. J., van der Zee, R., de Haan, C. A. M. \& Rottier, P. J. M. (2003) J. Virol. 77, 8801-8811.

19. Bosch, B. J., Martina, B. E., Van Der Zee, R., Lepault, J., Haijema, B. J., Versluis, C., Heck, A. J., De Groot, R., Osterhaus, A. D. \& Rottier, P. J. (2004) Proc. Natl. Acad. Sci. USA 101, 8455-8460.
SARS-CoV S2 trimeric HR1 constructs to be used as candidate vaccines against this virus.

Note Added in Proof. While this work was under review, a paper describing the 2.8 - $\AA$ resolution structure of a smaller fragment of the SARS-CoV S2 postfusion core was published (40).

We thank Dr. Winfried Weissenhorn and Dr. Micah Luftig for discussions. This work was supported in part by a grant from the Ministero dell'Istruzione, dell'Università e della Ricerca.

20. Liu, S., Xiao, G., Chen, Y., He, Y., Niu, J., Escalante, C. R., Xiong, H., Farmar, J., Debnath, A. K., Tien, P. \& Jiang, S. (2004) Lancet 363, 938-947.

21. Ingallinella, P., Bianchi, E., Finotto, M., Cantoni, G., Eckert, D. M., Supekar, V. M., Bruckmann, C., Carfi, A. \& Pessi, A. (2004) Proc. Natl. Acad. Sci. USA 101, 8709-8714.

22. Otwinowsky, Z. \& Minor, W. (1997) Methods Enzymol. 276, 307-326.

23. Brunger, A. T., Adams, P. D., Clore G. M., DeLano, W. L., Gros, P., Grosse-Kunstleve, R. W., Jiang J. S., Kuszewski, J., Niges, M., Pannu, N. S., et al. (1998) Acta Crystallogr. D 54, 905-921.

24. Jones, T. A., Zou, J. Y., Cowan, S. W. \& Kjeldgaard, M. (1991) Acta Crystallogr. A 47, 110-119.

25. Perrakis, A., Morris, R. \& Lamzin, V. S. (1999) Nat. Struct. Biol. 6, 458-463.

26. Collaborative Computational Project 4 (1994) Acta Crystallogr. D 50, 760-763.

27. Crick, F. (1953) Acta Crystallogr. A 6, 689-697.

28. Brown, J. H., Cohen, C. \& Parry, D. A. (1996) Proteins 26, 134-145.

29. Bullough, P. A., Hughson, F. M., Skehel, J. J. \& Wiley, D. C. (1994) Nature 371, 37-43.

30. Weissenhorn, W., Dessen, A., Harrison, S. C., Skehel, J. J. \& Wiley, D. C. (1997) Nature 387, 426-430.

31. Chan, D. C., Fass, D., Berger, J. M. \& Kim, P. S. (1997) Cell 89, 263-273

32. Baker, K. A., Dutch, R. E., Lamb, R. A. \& Jardetzky, T. S. (1999) Mol. Cell 3, 309-319.

33. Weissenhorn, W., Carfi, A., Lee, K. H., Skehel, J. J. \& Wiley, D. C. (1998) Mol. Cell 2, 605-616.

34. Malashkevich, V. N., Schneider, B. J., McNally, M. L., Milhollen, M. A., Pang, J. X. \& Kim, P. S. (1999) Proc. Natl. Acad. Sci. USA 96, 2662-2667.

35. Xu, Y., Liu, Y., Lou, Z., Qin, L., Li, X., Bai, Z., Pang, H., Tien, P., Gao, G. F. \& Rao, Z. (2004) J. Biol. Chem. 279, 30514-30522.

36. Park, H. E., Gruenke, J. A. \& White, J. M. (2003) Nat. Struct. Biol 10, $1048-1053$

37. Russell, C. J., Kantor, K. L., Jardetzky, T. S. \& Lamb, R. A. (2003) J. Cell Biol. 163, 363-374.

38. Sundaram, R., Lynch, M. P., Rawale, S. V., Sun, Y., Kazanji, M. \& Kaumaya, P. T. (2004) J. Biol. Chem. 279, 24141-24151.

39. Louis, J. M., Nesheiwat, I., Chang, L., Clore, G. M. \& Bewley, C. A. (2003) J. Biol. Chem. 278, 20278-20285.

40. Xu, Y., Lou, Z., Liu, Y., Pang, H., Tien, P., Gao, G. F. \& Rao, Z. (2004) J. Biol. Chem. 279, 49414-49419. 